Tropical Journal of Pharmaceutical Research February 2020; 19 (2): 421-425

ISSN: $1596-5996$ (print); 1596-9827 (electronic)

(c) Pharmacotherapy Group, Faculty of Pharmacy, University of Benin, Benin City, 300001 Nigeria.

Available online at http://www.tjpr.org

Original Research Article

http://dx.doi.org/10.4314/tjpr.v19i2.26

\title{
Comparison of the effects of Tripterygii totorum and sulfasalazine on rheumatoid arthritis: A retrospective cohort study
}

\author{
Renchun Huang ${ }^{1}$, Yongliang Tang ${ }^{2}$, Jiali Zeng ${ }^{3 *}$ \\ ${ }^{1}$ Department of Emergency, Hanzhong Central Hospital, Hanzhong, Shaanxi 723000, ${ }^{2}$ Department of Orthopedics, Xi'an \\ Central Hospital, Xi'an, Shaanxi 710003, ${ }^{3}$ Department of Critical Care Medicine, HanzhongPeople's Hospital, Hanzhong, \\ Shaanxi 723000, China
}

*For correspondence: Email: JimPeteeD@yahoo.com; Tel/Fax: 0086-0916-2706822

Sent for review: 20 August 2019

Revised accepted: 28 January 2020

\begin{abstract}
Purpose: To compare, in a retrospective study, the effects and safety profiles of Tripterygii totorum and sulfasalazine in patients with rheumatoid arthritis $(R A)$ following 24 weeks of treatment.

Methods: RA patients $(n=164)$ who were treated with Tripterygii totorum or sulfasalazine from August 2012 to February 2016 were included in this study. The major end-point was $\geq 20 \%$ improvement as per American College of Rheumatology (ACR) criterion (ACR 20 response) after 24 weeks. Moreover, ACR 50 and ACR 70 responses were studied. The safety parameters investigated comprised of adverse events, vital signs, as well as hematological and biochemical indices (blood counts, electrolyte levels, and kidney and liver function).

Results: At 24 weeks, ACR 20 response was $57.32 \%$ in patients on Tripterygii totorum, while the corresponding value in patients on sulfasalazine was $39.02 \%(p=0.02)$. In the Tripterygii totorum group, ACR 50 response was $41.46 \%$, while ACR 70 response was $29.27 \%$. In sulfasalazine group, ACR 50 response was identified in $26.83 \%$ of the patients, while ACR 70 response was seen in 21.95 $\%$ of patients. Adverse events were greater in the Tripterygii totorum group than in sulfasalazine group. Conclusion: These results suggest that Tripterygii Totorum significantly mitigates $R A$, with a tolerable safety profile. However, there is need for long-term or controlled trials to ascertain the therapeutic potential of Tripterygii totorum in RA.
\end{abstract}

Keywords: Traditional Chinese medicine, Tripterygii totorum, Sulfasalazine, Rheumatoid arthritis

This is an Open Access article that uses a fund-ing model which does not charge readers or their institutions for access and distributed under the terms of the Creative Commons Attribution License (http://creativecommons.org/licenses/by/4.0) and the Budapest Open Access Initiative (http://www.budapestopenaccessinitiative.org/read), which permit unrestricted use, distribution, and reproduction in any medium, provided the original work is properly credited.

Tropical Journal of Pharmaceutical Research is indexed by Science Citation Index (SciSearch), Scopus, International Pharmaceutical Abstract, Chemical Abstracts, Embase, Index Copernicus, EBSCO, African Index Medicus, JournalSeek, Journal Citation Reports/Science Edition, Directory of Open Access Journals (DOAJ), African Journal Online, Bioline International, Open-J-Gate and Pharmacy Abstracts

\section{INTRODUCTION}

Rheumatoid arthritis (RA) is a chronic and systemic autoimmune inflammatory disease of uncertain etiology which affects about $1 \%$ of human population. Usually, it results in disability and premature death. The management of RA involves pharmacological, non-pharmacological, invasive as well as surgical interventions. These treatments are often personalized, based on disorder manifestations such as physical activity, laboratory findings, symptoms, as well as prognostic indicators. Disease-modifying antirheumatic drugs (DMARDs) have been proposed 
as first-line therapy for RA, with immuneselective biologic agents as second-line anti-RA therapy. However, these drugs do not effectively provide therapy for RA. Thus, newer drugs are needed, in view of inadequate responses, adverse reactions, and increased cost of current treatments [1,2].

Although there are many DMARDS in China for RA therapy, traditional Chinese medicine (TCM) is preferred because it has been safely and effectively used for treating RA for more than 2000 years $[3,4]$. Tripterygii totorum is one of the commonest TCMs utilized in RA therapy. However, based on dearth of clinical trials, the actual safety and effectiveness of Tripterygii totorum in RA treatment is questionable. Moreover, there are no extant studies on comparison of the effectiveness of Tripterygii totorum with that of already-established allopathy RA therapy. The aim of present study was to compare the effectiveness and safety of Tripterygii totorum with those of sulfasalazine among RA patients treated for over 24 weeks. The endpoints included clinical as well as safety parameters.

\section{METHODS}

This retrospective observational study included RA patients treated with Tripterygii totorum or sulfasalazine from August 2012 to February 2016. Approval was received from the Institutional Review Board of Hanzhong Central Hospital (approval no. 201208245) and informed consent was received from participants. Patient confidentiality was strictly maintained. The study was conducted in line with Helsinki Declaration [5].

Patients aged greater than 18 years, and who had RA for a minimum of 1 year were included if they met the American College of Rheumatology (ACR) criterion for RA in functional classes I, II, or III $[6,7]$. Patients under steady doses of nonsteroidal anti-inflammatory drugs (NSAIDs) and/or corticosteroids (10 mg daily) for no less than one month prior to Tripterygii totorum or sulfasalazine administration were included. The exclusion criteria covered patients with other autoimmune diseases (arthritis, osteoarthritis or adjuvant arthritis lupus), serious disorder sof the kidney, cardiovascular system, liver, lung, and brain; pregnant, breastfeeding women, and women intending to get pregnant. The choice of treatment (TCM or allopathy) depended on the physician and the patient.
The major end-point used in this study was ACR 20 response at 24 weeks. To attain ACR 20, patients were expected to experience $\geq 20 \%$ improvement in tender and swollen joint counts, and $\geq 20 \%$ improvement in three or more of these criteria: patient's or physician's global health status assessment, pain assessment by patient on a visual analogue scale, function assessment by patient (utilizing a modified version of the Health Assessment Questionnaire [HAQ]), as well as serum C-reactive protein (CRP) level. Moreover, ACR 50 as well as ACR 70 responses were also calculated at 24 weeks. The safety parameters investigated comprised adverse events, vital signs, as well as hematological and biochemical indices (blood counts, electrolyte levels, and kidney and liver function assays). Safety assessment was done at baseline, and all through the treatment period. Medical chart reviews were done independently.

\section{Statistical analysis}

Values of categorical variables are expressed as numbers with percentages, while continuous variables are expressed as mean \pm standard deviation (SD). Student's $t$-test was used for analysis of continuous variables, while nonparametric tests were utilized for comparison of group differences. All statistical analyses were done utilizing SPSS version 21.0 (SPSS Inc, Chicago). Values of $p<0.05$ were taken as indicative of statistical significance.

\section{RESULTS}

The clinical/demographic characteristics of the patients included in the study are presented in Table 1. Of the 164 patients, 82 were on Tripterygii totorum, while 82 patients were on sulfasalazine. Although the baseline characteristics were similar in both groups, there were significant differences in age $(p=0.034)$ and RA duration $(p<0.001)$ among the older patients, and longer RA duration in the Tripterygii totorum group.

The results of summaries with respect to ACR at 24 weeks are shown in Table 2. Post 24-week therapy, $57.32 \%$ of patients given Tripterygii totorum and $39.02 \%$ of patients who received sulfasalazine experienced $\geq 20 \%$ improvement in activity disorder, as shown in ACR20 response $(p=0.02)$. Comparable improvements with respect to ACR 50 and ACR 70 responses were observed. In Tripterygii totorum group, ACR 50 response was identified in $41.46 \%$ of $\mathrm{RA}$ patients, while ACR 70 response was seen in $29.27 \%$ of RA patients. 
Table 1: Clinical/demographic characteristics of patients

\begin{tabular}{|c|c|c|}
\hline Characteristics & TT group & SZ group \\
\hline No. of patients & 82 & 82 \\
\hline Male, $\mathrm{n}(\%)$ & $18(21.95 \%)$ & $16(19.51 \%)$ \\
\hline Age (years)* & $51.83(9.42)$ & $49.75(10.88)^{\star \star}$ \\
\hline RA duration & $74.37(75.16)$ & $61.85(63.94)^{* \star}$ \\
\hline$\leq 24$ months & $32(39.02 \%)$ & $38(46.34 \%)$ \\
\hline$>24$ months & $50(60.98 \%)$ & $44(53.66 \%)$ \\
\hline Positive RF & $64(78.05 \%)$ & $68(82.93 \%)$ \\
\hline Joint tenderness scores (total) & $21.34(8.27)$ & $17.58(9.93)$ \\
\hline Joint swelling scores (total) & $10.22(5.41)$ & $10.89(5.82)$ \\
\hline Joint stiffness (morning, min) & $88.03(69.29)$ & $93.74(77.18)$ \\
\hline Patient's global assessment (0-100) & $68.62(17.94)$ & $67.44(17.14)$ \\
\hline Physician's global assessment (0-100) & $67.51(16.39)$ & $66.86(16.48)$ \\
\hline $\mathrm{CRP}(\mathrm{mg} / \mathrm{L})$ & $19.16(4.67)$ & $17.42(3.26)$ \\
\hline $\mathrm{ESR}(\mathrm{mm} / \mathrm{h})$ & $47.93(29.69)$ & $45.47(26.11)$ \\
\hline $\lg G(g / L)$ & $17.83(7.12)$ & $17.38(5.64)$ \\
\hline $\lg A(g / L)$ & $2.98(1.85)$ & $3.07(1.61)$ \\
\hline $\operatorname{lgM}(g / L)$ & $1.92(0.81)$ & $2.13(0.79)$ \\
\hline
\end{tabular}

$\mathrm{CRP}=$ C-reactive protein; ESR = erythrocyte sedimentation rate; RA = rheumatoid arthritis; RF = rheumatoid factor; SZ = sulfasalazine; TT = Tripterygii totorum; *Values are expressed as mean, with standard deviation in bracket, unless indicated otherwise; ${ }^{* *} p<0.05$, between TT group and SZ group

Table 2: Outcome of ACR summary at 24 weeks

\begin{tabular}{llll}
\hline Outcome & $\begin{array}{l}\text { TT group, } \\
\text { n(\%) }\end{array}$ & $\begin{array}{l}\text { SZ group, } \\
\mathbf{n}(\%)\end{array}$ & $\boldsymbol{P}$ \\
\hline ACR20 & $47(57.32 \%)$ & $32(39.02 \%)$ & $0.02^{*}$ \\
ACR50 & $34(41.46 \%)$ & $24(29.27 \%)$ & $<0.01^{*}$ \\
ACR70 & $22(26.83 \%)$ & $18(21.95 \%)$ & 0.748 \\
\hline
\end{tabular}

$\mathrm{ACR}=$ American College of Rheumatology; $\mathrm{SZ}=$ Sulfasalazine; TT = Tripterygii totorum

In the sulfasalazine group, ACR 50 response was identified in $26.83 \%$ of patients, while ACR 70 response was seen in $21.95 \%$ of patients. There was a significant difference in ACR50 responses between the two groups $(p<0.01)$. Data on adverse events are presented in Table 3. The extent of adverse events was greater in the Tripterygii totorum group than in sulfasalazine group. Overall, the most widely observed adverse event in Tripterygii totorum group was diarrhea (21 cases, $25.61 \%)$, followed by dyspepsia (20 cases, $24.39 \%)$, nausea (8 cases, $21.95 \%$ ), and abdominal pain (15 cases, $18.29 \%$ ), all of which were related to the gastrointestinal system. The least reported adverse events ( 1 case, 1.22\%) involved pneumonia, deep vein thrombosis, and neutropenia. The adverse events reported in majority of patients who took sulfasalazine were nausea (24 cases, $29.27 \%$ ), followed by diarrhea (16 cases, $19.51 \%)$, vomiting (14 cases, $17.07 \%$ ), and headache (14 cases, 17.07 $\%$ ). The least reported adverse event (1 case, $1.22 \%$ ) involved gastric bleeding and peripheral edema.

Table 3: Adverse events

\begin{tabular}{llll}
\hline Adverse event & \multicolumn{1}{c}{ TT group, $\mathbf{n}(\%)$} & SZ group, $\mathbf{n}(\%)$ & P-value \\
\hline Nausea & $18(21.95)$ & $24(29.27)$ & 0.546 \\
Vomiting & $14(17.07)$ & $14(17.07)$ & 0.388 \\
Diarrhea & $21(25.61)$ & $16(19.51)$ & 0.231 \\
Abdominal pain & $15(18.29)$ & $9(10.98)$ & 0.073 \\
Dyspepsia & $20(24.39)$ & $8(9.76)$ & 0.557 \\
Gastric bleeding & 0 & $1(1.22)$ & 0.062 \\
Pneumonia & $1(1.22)$ & 0 & 0.686 \\
Influenza & $2(2.44)$ & $3(3.66)$ & 0.093 \\
Headache & $9(10.98)$ & $14(17.07)$ & 0.224 \\
Rash & $10(12.19)$ & $8(9.76)$ & 0.085 \\
Peripheral edema & $3(3.66)$ & $1(1.22)$ & 0.569 \\
Cough & $3(3.66)$ & $2(2.44)$ & 0.778 \\
Blurred vision & $5(6.1)$ & 0 & 0.056 \\
Hot flush & $5(6.1)$ & $2(2.44)$ & 0.264 \\
Amenorrhea & $9(10.98)$ & $3(3.66)$ & 0.092 \\
Anemia & 0 & $5(6.1)$ & 0.077 \\
Deep venous thrombosis & $1(1.22)$ & 0 & 0.267 \\
Neutropenia & $1(1.22$ & 0 & 0.183 \\
\hline
\end{tabular}

$\mathrm{SZ}=$ Sulfasalazine; TT $=$ Tripterygii totorum 


\section{DISCUSSION}

The results of this retrospective observational study demonstrate that Tripterygii totorum, which is utilized in TCM, is capable of effectively treating RA. Within 24 weeks, therapy using Tripterygii totorum resulted in greater reductions in symptoms and signs of RA than treatment with sulfasalazine. Moreover, the ACR20 and ACR50 responses due to Tripterygii totorum were significantly better, when compared with those of sulfasalazine. Tripterygii totorum tablets were used at maximum dose of $10 \mathrm{mg}$ thrice daily, while sulfasalazine was used at maximum dose of $2 \mathrm{~g}$ per day. Since Tripterygii totorum was administered orally, the oral RA drug i.e. sulfasalazine was used for comparison. Sulfasalazine has been used as a comparator in several studies and was shown to be comparable to oral DMARDS [8-11]. Before initiating therapy, the patient was given an option to choose between TCM and allopathy. Personal satisfaction was higher with TCM than sulfasalazine due to the closeness of the patients with Chinese history.

Although the included patient population used in this study was similar to those in a few studies with reference to activity disorder and joint damage $[8,12,13]$, the results obtained here are more encouraging. However, there is need to confirm the results using controlled trials with large cohorts.

Gastrointestinal system-associated adverse events were seen in RA patients during the initial course of therapy with Tripterygii totorum and sulfasalazine. However, the adverse events abated more in patients who received Tripterygii totorum than those treated with sulfasalazine. Smaller drug dosage at treatment initiation or progressive increase in dose to maximum levels might make the drug more tolerable, in addition to counselling patients on the need to sustain the treatment by taking their drugs regularly. None of the adverse events was deemed serious.

\section{Limitations of the study}

Since this study is an observational investigation, the effectiveness of therapy might be affected by the patient's or physician's decisions in real life setting. In such scenario, selection bias is a possibility. The experience of adverse events may not have been reported in full (underreporting). The association of adverse events with drugs might be inconsistent due to the involvement of different physicians. The time span of 24 weeks might not have provided information on long-term effect. Due to retrospective nature, only patients with 24-week data were included, thereby excluding information on drug withdrawals and attrition. The present study has missing data, a major concern if the data is informative. Thus, a comprehensive conclusion should be made with caution in view of the potential effect of missing data. Moreover, this study was based on a single-center with restricted number of patients. Therefore, generalization of the results should be made with caution.

\section{CONCLUSION}

The study outcomes suggest that Tripterygii totorum produces statistically significant RA improvement with tolerable safety profile. However, there is need for controlled, multicenter, larger-sample trials to determine the longterm therapeutic effect of Tripterygii totorum.

\section{DECLARATIONS}

\section{Conflict of interest}

No conflict of interest is associated with this work.

\section{Contribution of authors}

We declare that this work was done by the authors named in this article and all liabilities pertaining to claims relating to the content of this article will be borne by the authors. Renchun Huang drafted this manuscript with the help of Yongliang Tang under supervision of Jiali Zeng. Both Renchun Huang and Yongliang Tang collected data and did statistical analysis.

\section{Open Access}

This is an Open Access article that uses a funding model which does not charge readers or their institutions for access and distributed under the terms of the Creative Commons Attribution License (http://creativecommons.org/licenses/by/ 4.0) and the Budapest Open Access Initiative (http://www.budapestopenaccessinitiative.org/rea d), which permit unrestricted use, distribution, and reproduction in any medium, provided the original work is properly credited.

\section{REFERENCES}

1. Fanet-Goguet $M$, Martin $S$, Fernandez $C$, Fautrel $B$, Bourgeois $P$. Focus on biological agents in rheumatoid 
arthritis: newer treatments and therapeutic strategies. Therapie 2004; 59: 451-461

2. O'Dell JR. Therapeutic strategies for rheumatoid arthritis. $N$ Engl J Med 2004; 350: 2591-2602, doi:10.1056/NEJMra040226

3. $L i Y L$. The advance on treating $R A$ with traditional Chinese medicine. J Guangxi Trad Chin Med Univ 2004; 7(3):81-83

4. Fu ZT, Huang $W X$, Yuan J. Clinical research progress of $R A$ treated by Chinese medicine. Hunan J Trad Chin Med 2005; 21(4): 93-95.

5. World Health Organization. Declaration of Helsinki. BrMed J 1996; 313(7070): 1448-1449.

6. Felson DT, Anderson JJ, Boers M, Bombardier C, Furst $D$, Goldsmith $C$, Katz LM, Lightfoot $R$ Jr, Paulus $H$, Strand $V$, et al. American College of Rheumatology. Preliminary definition of improvement in rheumatoid arthritis. Arthritis Rheum 1995; 38: 727-735.

7. Cader MZ, Filer A, Hazlehurst J, de Pablo P, Buckley CD, Raza K. Performance of the 2010 ACR/EULAR criteria for rheumatoid arthritis: comparison with 1987 ACR criteria in a very early synovitis cohort. Ann Rheum Dis 2011; 70(6): 949-55.

8. Goldbach-Mansky R, Wilson M, Fleischmann R, Olsen N, Silverfield J, Kempf $P$, Kivitz A, Sherrer $Y$, Pucino F, Csako $G$, et al. Comparison of Tripterygium wilfordii Hook $F$ versus sulfasalazine in the treatment of rheumatoid arthritis: A randomized trial. Ann. Intern. Med 2009;151: 229-240.

9. Suarez-Almazor ME, Belseck E, Shea B, Wells G, Tugwell $P$. Sulfasalazine for rheumatoid arthritis.
Cochrane Database Syst Rev 2000 CD000958 [PMID: 10796400].

10. Smolen JS, Kalden JR, Scott DL, Rozman B, Kvien TK, Larsen A, Loew-Friedrich I, Oed C, Rosenburg $R$. Efficacy and safety of leflunomide compared with placebo and sulphasalazine in active rheumatoid arthritis: a double-blind, randomised, multicentre trial. European Leflunomide Study Group. Lancet 1999; 353: 259-266. [PMID: 9929017]. [PubMed: 9929017]

11. Proudman SM, Conaghan PG, Richardson $C$, Griffiths $B$, Green MJ, McGonagle D, Wakefield RJ, Reece RJ, Miles $S$, Adebajo A, et al. Treatment of poor-prognosis early rheumatoid arthritis. A randomized study of treatment with methotrexate, cyclosporin $A$, and intraarticular corticosteroids compared with sulfasalazine alone. Arthritis Rheum 2000; 43: 18091819. [PMID: 10943871].

12. Cohen S, Cannon GW, Schiff M, Weaver A, Fox R, Olsen $N$, Furst D, Sharp J, Moreland L, Caldwell J, et al. Twoyear, blinded, randomized, controlled trial of treatment of active rheumatoid arthritis with leflunomide compared with methotrexate. Utilization of Leflunomide in the Treatment of Rheumatoid Arthritis Trial Investigator Group. Arthritis Rheum 2001; 44: 1984-1992. [PMID: 11592358].

13. Kremer JM, Genant HK, Moreland LW, Russell AS, Emery P, Abud-Mendoza C, Szechinski J, Li T, Ge Z, Becker JC, et al. Effects of abatacept in patients with methotrexate-resistant active rheumatoid arthritis: a randomized trial. Ann Intern Med 2006; 144: 865-876. [PMID: 16785475]. 